\title{
Patient-safety reforms inhibited by systemic impediments
}

$\mathrm{T}$ he progress of the patient safety movement is being stymied by regulatory, structural and attitudinal problems, according to speakers at the eighth annual Canadian Healthcare Safety Symposium.

"Our own safety is on the line," warned Dr. Dennis Kendel, chief executive officer of the College of Physicians and Surgeons of Saskatchewan, noting that health care providers are sometimes also patients.

Nurse John Lewis told the Oct. 2325 conference in Winnipeg, Man. that his 11-year-old daughter Claire's postoperative death 8 years ago was "utterly and completely preventable."

At her bedside, he had known that something was wrong, and documented events. She died as a direct result of mismanagement of fluids and electrolytes and her death led Lewis to successfully campaign to have the hospital and health care providers held to account for their errors.

But Lewis' success was an anomaly. The patient-safety advocate said he spoke to many families that have fought "all in vain" for responsibility and accountability.

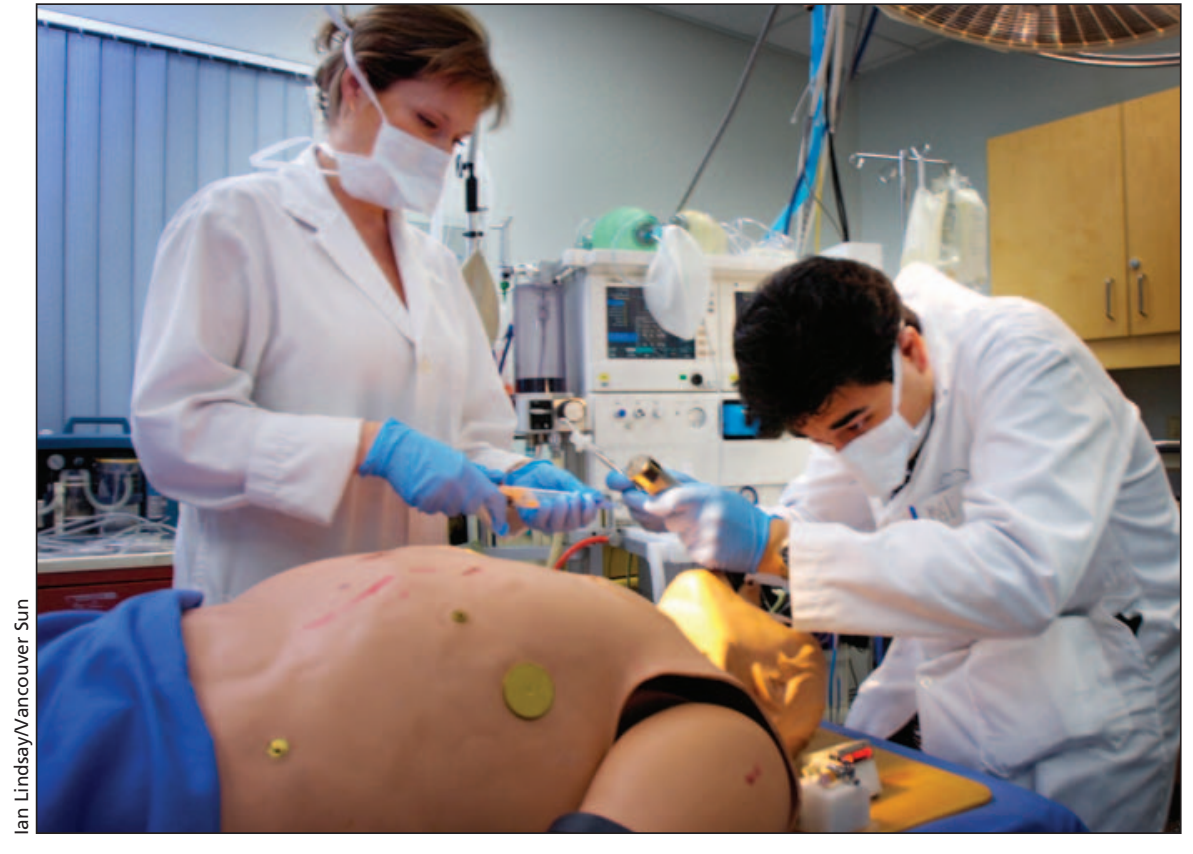

In an experiment using patient simulator technology, medical residents toiling for 24 consecutive hours were found to make twice as many major errors (see page 1254).

"What makes the health care system so unique that it doesn't realize when something is wrong?" asked Lewis, author of Beware the Grieving Warrior. "Why is health care seem- ingly exempt from concepts in other high-risk industries?"

The push to create a "safety culture" in health care is important, but it may be unproductive unless it is accompanied

\section{Improved double-checking reduces — but doesn't eliminate - medication errors}

Deadly medication errors can be reduced with refinements to protocols for independent double-checking of high-risk medication, according to research conducted at the University Health Network in Toronto.

A multidisciplinary team of researchers used observation and laboratory simulations to study the flow of tasks involved with checking ambulatory infusion pumps in chemotherapy outpatient clinics.

A significant reduction in errors related to patient identification and order-label mismatch resulted from the use of a revised and more specific checklist, tested by the researchers, which took the same length of time to complete as the previous standard form.

However, the new checklist did not reduce pump programming errors. The highly publicized 2006 death of a 43year-old Canadian woman resulted from a calculation error in pump programming, as a result of which she received, in
4 hours, 4 days' worth of the drug fluorouracil from her infusion pump.

The research indicated that neither the old nor the new checklist helped prevent clinically inappropriate orders (for example, if a doctor ordered 10 times the usual dose of a medication and the pharmacist failed to notice the mistake).

Forms are useful for mechanistic checks, but do not help health care workers "think big," said biomedical engineer Tony Easty.

Double-checking is not fail safe and should not be completely relied upon, but specific checklists can result in better detection of errors, Easty noted.

The research, funded by the Canadian Patient Safety Institute, has been submitted for publication.

Identifiable medication errors accounted for $7.1 \%$ of all cases of serious injury reported to the Institute for Safe Medication Practices for the first quarter of 2008. 
by a regulatory system, warned Jonathan Aleck of Australia's Civil Aviation Safety Authority.

In the high-risk aviation industry, the key regulatory functions are to develop standards and rules, conduct surveillance, monitor performance to identify trends and risks, and develop enforcement strategies to secure compliance, said Aleck, head of the authority's legal services group.

But in health care, standards when they exist - are not always followed, poor behaviour is tolerated, there are few rules to violate, and there is little supervision of work practices, Bill Runciman, president of the Australian Patient Safety Foundation, told the symposium.

Indeed, for many medical conditions — including coronary artery disease, sexually transmitted diseases and asthma patients receive the recommended care and treatment, on average only about half the time, he said. "There is a staggering amount of data that show we're doing the wrong job. ... We need better responsive regulation." Runciman argued that health care professionals need user-created tools that are developed through close attention to workflow, where processes are documented, auditing is easy, and improvements can readily be made as new evidence becomes available.

Instead, there's a tendency to criticize evidence in order to maintain the status quo, he said, arguing that medical practice is currently dictated by traditional approaches and "collective mindlines."

This tendency among physicians to share a way of thinking made objective oversight of forensic pathologist Dr. Charles Smith very difficult, said Justice Stephen Goudge, who headed the province of Ontario's recently completed Inquiry into Pediatric Forensic Pathology, which examined errors Smith made in roughly 20 cases, 12 of which led to wrongful criminal convictions of parents (CMAJ 2008;179[10]:995).

Smith and his superiors shared an inclination to take a "think dirty" approach to child injuries and automatically suspect abuse, rather than to "think truth," Goudge said. As a result, there was "completely inadequate" oversight of his activities.

Sir Ian Kennedy, now chair of the
United Kingdom Healthcare Commission, headed an inquiry into pediatric cardiac surgery. Many of his recommendations were acted on, but he identified several impediments to change: a lack of incentives to report and learn from errors, poor teamwork, the low priority given by management and government to safe patient care, a culture of "blame and shame" and litigation for clinical negligence.

When patients have to litigate to obtain compensation for injury, the dis satisfaction of the parties is virtually guaranteed because respect for individuals has been undermined, said Dr. Rob Robson, chief patient-safety officer with the Winnipeg Regional Health Authority. "Litigation is in some ways a guarantee of nonlearning."

The existence of silos in medicine is another impediment to improving patient safety, said Kendel. The fact that health care professionals are "regulated in silos" means there are few mechanisms to "allow, let alone encourage" teamwork.

"The structures cry out for change. ... We have to build structures to have

\section{Sleepy medical residents make major errors tackling complex problems, study finds}

After 24 hours of wakefulness, medical residents made twice as many major errors tackling complex clinical problems as they did at the beginning of a session during which they performed tasks in controlled experimental conditions using patient simulator technology.

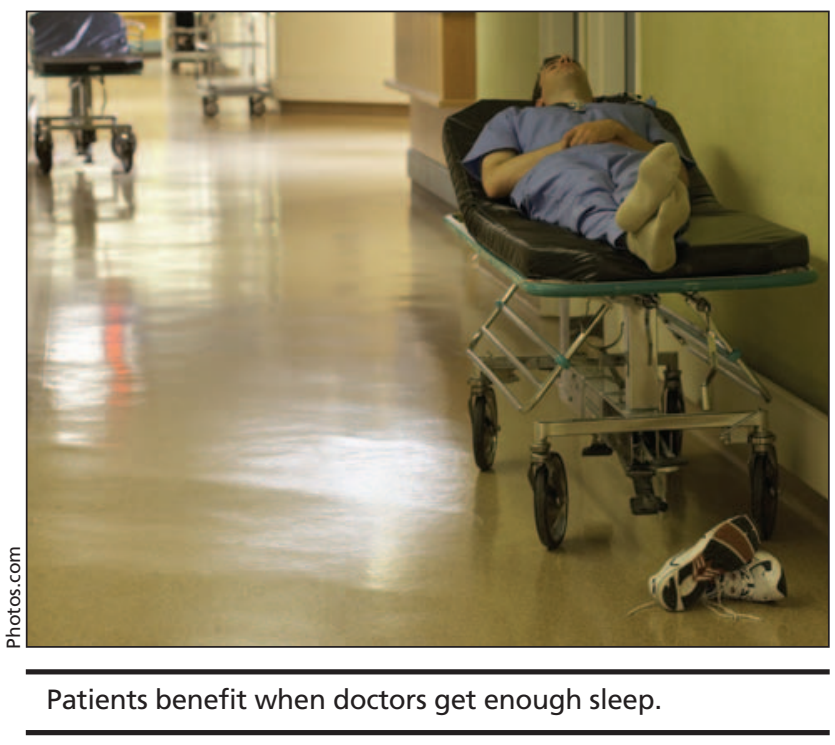

The research, led by Dr. Najib Ayas of the sleep disorders program at the University of British Columbia, was funded by the Canadian Patient Safety Institute. It has not been published.

Scorers blinded to the time of the session reviewed videotapes of the performance of 12 residents over a 26hour period. The doctors actually improved their handling of straightforward cardiac arrests over the session, but their handling of complex emergencies - anaphylaxis, postoperative major pulmonary embolism, meningococcemia and status epilepticus - worsened.

Errors included failure to give steroids, epinephrine or antihistamine for anaphylaxis; failure to give heparin for pulmonary embolism; failure to give dilantin for status epilepticus; and failure to intubate for respiratory failure.

There are no national guidelines on hours of work for medical residents, said Ayas, who deemed the current practice of having medical residents on call for 24 hours "unacceptable."

Since medical residents are paid by the year, not by the hour, cost may be a key barrier to implementing reasonable work-hour limits, he said. 'I'm a sleep guy and I think sleep is important. ... But in this society, people tend to brag about how little they sleep." 
physicians accountable to other [health care] team members," he said, noting that most doctors are independent contractors, while other health care professionals are typically employees.

Kendel also noted that in the UK, electronic technology in the offices of general practitioners not only supports decision-making but provides regular feedback to physicians about their practice. Canada's slow progress compared to other countries in embracing information technology and electronic health records is "embarrassing," he said.

Saskatchewan has a pharmacy information program that allows any doctor to $\log$ on and view the medication profile for the patient "sitting in front of them." But Kendel said many doctors don't have computers in their examining rooms and use of the program is discouragingly low. - Ann Silversides, CMAJ

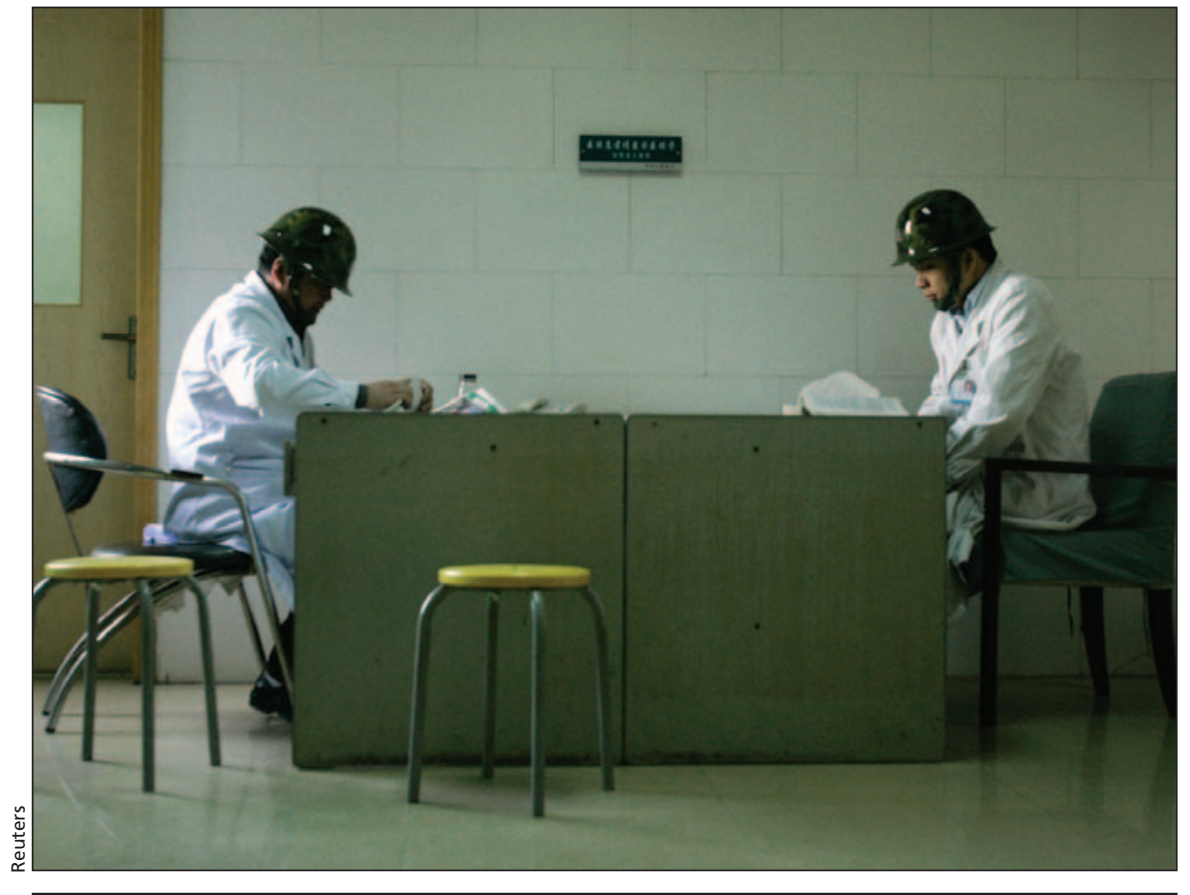

Doctors wear hard hats during their rounds after coming under attack from relatives seeking compensation over a patient's death at Shanxia Hospital in Shenzhen, China.

\section{Only about $2 \%$ of injured patients receive compensation, law professor says}

A "huge gulf" exists between the number of Canadian patients injured by negligence and those who receive compensation, says University of Alberta law professor Gerald Robertson. "One must seriously question the efficacy of a model which compensates so few who are entitled to it."

"What is clear and almost irrefutable is that the present system is not working," he said, asking delegates "whose institutional responsibility should it be to look at this and say the time has come to give serious thought to change?"

Doctors who have been sued report feelings of anger, distress and being personally attacked, and "a significant number" report a desire to abandon the practice of medicine, said Robertson, in his talk, "The Emotional and Financial Impact of Medical Malpractice Litigation."

Only about $2 \%$ of patients injured by negligence in Canada receive compensation, he said, basing his calculation on figures from the Canadian Medical Protective Association, research estimating the number of preventable adverse events in Canadian hospitals (CMAJ 2004;170: 1687-8), as well as studies from the United States.

Meanwhile, the number of lawsuits against Canadian doctors is dropping — down 30\% since 1998 (from 1339 commenced in 1998 to 928 commenced in 2007) - and only $30 \%$ of plaintiffs seeking compensation are successful in court, he noted.

Medical negligence cases are complex, time-consuming, expensive and almost always undertaken on a contingency-fee basis. As a result, lawyers are unlikely to take on cases unless there is a chance of a settlement valued over $\$ 100000$, he said, noting that lawyers usually seek a fee equal to $30 \%$ of a successful settlement.

The patient-safety movement may "raise consciousness" about the need for better compensation for patients, since it will likely raise awareness about the frequency of adverse medical events, Robertson later said in an interview. But many injured patients reportedly seek not money but rather an acknowledgement of what happened, assurances that other patients won't suffer the same harm, and an apology.

As a result, the patient safety movement's success at promoting disclosure and apologies may well diminish the number of lawsuits, but the problem of injured patients not receiving compensation will remain.

Saskatchewan lawyer Reg Watson took issue with Robertson's figures, arguing that many injured patients do receive compensation directly from hospitals, through the Healthcare Insurance Reciprocal of Canada and from insurers other than the Canadian Medical Protective Association.

Justice Murray Sinclair, who conducted an inquiry into the deaths of 12 babies during heart surgery in a Winnipeg hospital, told an earlier session he favoured no-fault insurance since it would encourage a greater recognition and acknowledgement of errors. But the issue of punishment could not be satisfied through a no-fault approach, Sinclair said. 